\title{
Fast-electron refluxing effects on anisotropic hard-x-ray emission from intense laser-plasma interactions
}

\author{
K. McKeever, M. Makita, G. Nersisyan, T. Dzelzainis, S. White, B. Kettle, B. Dromey, \\ M. Zepf, G. Sarri, D. Doria, H. Ahmed, C. L. S. Lewis, and D. Riley* \\ Centre for Plasma Physics, School of Mathematics and Physics, Queen's University Belfast, University Road, \\ Belfast BT7 1NN, United Kingdom
}

A. P. L. Robinson

Central Laser Facility, Rutherford-Appleton Laboratory, Chilton, Didcot OX11 OQX, United Kingdom

(Received 17 May 2014; revised manuscript received 3 February 2015; published 16 March 2015)

\begin{abstract}
Fast-electron generation and dynamics, including electron refluxing, is at the core of understanding high-intensity laser-plasma interactions. This field is itself of strong relevance to fast ignition fusion and the development of new short-pulse, intense, $\mathrm{x}$-ray, $\gamma$-ray, and particle sources. In this paper, we describe experiments that explicitly link fast-electron refluxing and anisotropy in hard-x-ray emission. We find the anisotropy in $\mathrm{x}$-ray emission to be strongly correlated to the suppression of refluxing. In contrast to some previous work, the peak of emission is directly along the rear normal to the target rather than along either the incident laser direction or the specular reflection direction.
\end{abstract}

DOI: 10.1103/PhysRevE.91.033107

PACS number(s): 52.38.Ph, 52.38.Dx, 52.70.La

In high-intensity laser-plasma interactions, it is common to generate a population of so called fast (or hot) electrons via absorption mechanisms such as resonance absorption and $\mathbf{J} \times \mathbf{B}$ acceleration [1]. Typical fast-electron energies range from several tens of $\mathrm{keV}$ to several $\mathrm{MeV}$ [2-7] depending on the intensity of the laser and the wavelength. It is common to characterize them by an effective temperature even if they do not strictly follow a Maxwellian distribution. This effective temperature is usually greatly in excess of the so called "thermal" temperature of the plasma that may arise as a result of collisional absorption processes such as inverse bremsstrahlung. The dynamics of fast electrons as they penetrate a solid density target are of great interest, partly due to their relevance to fast ignition fusion schemes and partly due to the central role of electron dynamics in the development of laser-based ion acceleration schemes [8] and x-ray and $\gamma$-ray sources that can be used in a variety of scientific applications [9-12]. A phenomenon that has been previously observed is the refluxing of electrons. In this process, fast electrons created from the laser plasma interaction can travel through the foil until they reach the rear, where space-charge effects prevent most of them leaving the foil but pull them back into the foil after a change of direction. A small number of very energetic electrons will escape the target until space charge prevents further losses. This depends on the size and geometry of the target as well as irradiation conditions. The efficiency of refluxing has been discussed by Myatt et al. [13], who present estimates based on a capacitive model for picosecond irradiation at $1.06 \mu \mathrm{m}$. For our conditions with $\mathrm{mm}$ sized targets and $2 \times 10^{18} \mathrm{~W} \mathrm{~cm}^{-2}$ irradiance (see below), we expect in excess of $99 \%$ efficiency, similar to that assumed by Quinn et al. [14]. In the case of bare foils, a retarding electric field caused by the escape of charge pulls back electrons. When a dielectric layer is added, this retarding electric field

${ }^{*}$ Corresponding author: d.riley@qub.ac.uk penetrates into the dielectric and causes electrical breakdown, as discussed by Tikhonchuk [15]. This breakdown dissipates energy, and a return current can neutralize the fast electron current, thus allowing fast electrons to be deposited in the dielectric layer and not be refluxed to the metallic foil. The refluxing process has previously been observed via its effect on $K-\alpha$ radiation emission $[13,16,17]$ and also as transverse refluxing from target edges in limited area targets [18]. Another important characteristic of intense laser-plasma interaction, seen at high intensity, is the anisotropy in hard-x-ray emission that can result from beamlike behavior of fast electrons, e.g., $[19,20]$.

In this paper, we discuss the coupling of these two effects through the observation of hard-x-ray emission that changes from isotropic to anisotropic as we introduce a layer of epoxy to the rear of the laser-irradiated foils. The observations are made at irradiances in the relativistic regime. The experiment was carried out with the high-power laser system TARANIS [21], situated at Queen's University Belfast. This Nd:glass chirpedpulse-amplified laser provides pulses of 800 fs full width at half-maximum (FWHM) duration at $1.053 \mu \mathrm{m}$ wavelength. The amplified spontaneous emission (ASE) intensity contrast of the laser at $2 \mathrm{~ns}$ before the main pulse was measured to be $10^{-7}$. The prepulse activity consisted of a few picosecond duration prepulses at up to approximately $2.4 \mathrm{~ns}$ ahead of the main pulse, with an intensity contrast of $2 \times 10^{-7}$ compared with the main pulse. The $p$-polarized beam was focused by an F/3.3 off-axis parabola (OAP) to a focal spot of $12 \mu \mathrm{m}$ FWHM diameter containing $50 \%$ of the energy [22]. For this experiment, we estimate a peak intensity of just under $2 \times$ $10^{18} \mathrm{~W} \mathrm{~cm}^{-2}$ (normalized vector potential $a_{0} \sim 1.3$ ) for $45^{\circ}$ incidence on target for a $5 \mathrm{~J}$ shot. Targets were foils of Ti that were either bare or backed by a thick $(\sim 1 \mathrm{~mm})$ layer of epoxy (A/epichlorohydrin, $\mathrm{C}_{21} \mathrm{H}_{25} \mathrm{ClO}_{5}$ ).

A schematic of our experiment is shown in Fig. 1(a). We fielded simple but robust instruments that measured the bremsstrahlung radiation generated by interaction of the fast 


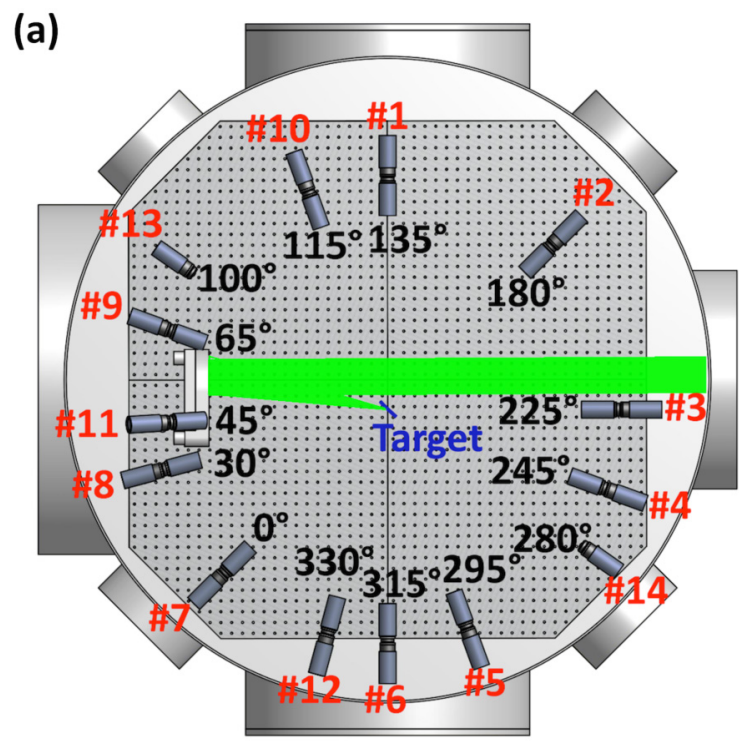

(b)

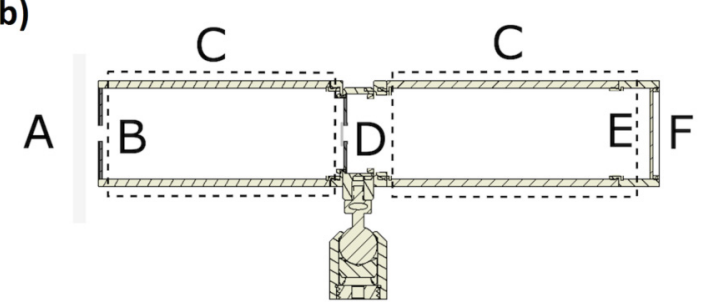

FIG. 1. (Color online) (a) Layout of the experiment showing the relative positions of the detectors to the laser pulse direction. The fronts of the detectors are all $440 \mathrm{~mm}$ from the target with the image plate $590 \mathrm{~mm}$ from the target. (b) Sketch of the detector construction. $A$ is the polystyrene layer, $B$ is the lead collimating slit, $C$ is lead shielding around the C-tube, $D$ is the lead filter array. $E$ is the IP sample and $F$ is a removable lead shielding disc. The dashed lines show the placement of the outer Pb shielding. The total length of the instrument is $150 \mathrm{~mm}$.

electrons with the foil. These are shown in the cross section in Fig. 1(b). The main bodies of the detectors were constructed from sections of aluminum lens tubing. A front disk of 1 -mm-thick lead with a slit measuring $25 \times 5 \mathrm{~mm}$ acted as a collimator, which limited signal measurement to the region directly in line with the detector. Strips of lead were used to create a 12-filter array in the middle with thicknesses from 50 to $325 \mu \mathrm{m}$. A piece of image plate (IP) was placed in a short, removable piece of tubing at the end, $7.5 \mathrm{~cm}$ away from the filters, to reduce fluorescence signal from the filters. The instruments were surrounded by shielding made of a 1.8-mm-thick lead sheet; a $30 \mathrm{~mm}$ diameter lead disk made of the same material was placed in the rear of the removable lens tube section to protect the IP sample from exposure to the rear. Comparison of the signal through the different filters of this array allowed us to estimate the effective fast-electron temperature by making some plausible assumptions about the bremsstrahlung spectrum.

Up to 14 such detectors were placed at a total of 15 positions in the horizontal plane all around the target with the image plate $590 \mathrm{~mm}$ from the target. An exception was detector 11, which was slightly above the horizontal plane $\left(21^{\circ}\right)$ to look past a mirror mount. Most shots used 12 detectors, but sometimes two additional detectors with a six-filter array (50-300 $\mu \mathrm{m} \mathrm{Pb}$ ) were deployed. Between the targets and the detectors were 3-mm-thick layers of polystyrene. This is thick enough to be beyond the range of all electrons below $700 \mathrm{keV}$ but still virtually transparent to all photons detectable through the lead filters. Previous work with a single detector [22] had indicated that resonance absorption was the dominant absorption mechanism and that the fast electron temperatures were below $100 \mathrm{keV}$ for our conditions, and so very few fast electrons could reach the detectors to cause fluorescence. Using scaling arguments [23] and estimates of the number of electrons escaping [13], we estimate that bremsstrahlung caused by electrons hitting the polystyrene accounts for less than $1 \%$ of the signal at the image plate and is negligible in terms of signal to noise for all filter channels.

The image plate used $[24,25]$ has been calibrated out to $662 \mathrm{keV}$. By measuring the signal levels in mPSL (PSL denotes photostimulation level) through the different filters and assuming an effectively Maxwellian electron distribution as an approximation, we can not only estimate the total hard-x-ray conversion efficiency $\left[(1-1.5) \times 10^{-4}\right]$ but we can also infer the effective electron temperature from the ratio of signals through the known filter thicknesses.

Before we discuss the results of our experiment, it may be of interest to see what level of anisotropy is expected in the bremsstrahlung $\mathrm{x}$-ray signal. We expect, as seen below and from earlier work [22], to have fast-electron temperatures of around $60 \mathrm{keV}$. This is only a fraction of the mass energy but still leads to an electron speed of $\sim 0.45 \mathrm{c}$. We can see in Fig. 2 that we do indeed expect a strong anisotropy even when considering the lowest energy of detected photons and typical electron energies.

In Fig. 3 we can see a typical raw image for detector 8 placed $30^{\circ}$ from the front normal of the target. We show data from a Ta target for comparison because the higher level of emission allows us to more clearly see the filter boundaries, and this helps in the analysis. We did not use Ta generally for this work as this would introduce opacity effects for the thicker targets, which would complicate the analysis. We can also see in Fig. 3 an averaged line out for one of the rows of filters, showing the signal uncorrected for background. We can see that the image plates have a general background level of a few mPSL that is fairly uniform and easily removed from the data. This background is only there when data are taken and so probably originates from fluorescence. A study of the fluorescence [26] using a modified detector design indicated that the background dropped with a filter-image plate distance up to about $50 \mathrm{~mm}$ but it did not change beyond this, indicating that we minimized the background and that fluorescence from the chamber and detector body may be responsible.

In Fig. 4(a), we can see a typical comparison of a background-subtracted signal through the filters with a prediction for a single effective Maxwellian fast-electron temperature of $62 \mathrm{keV}$. The error bars in this figure simply represent the standard deviation in the averaged line out, as seen in Fig. 3(b). Error bars in quantities such as angularly resolved emission profile and temperature, derived below, are calculated from statistical analysis of multiple shots. As noted above, it is 

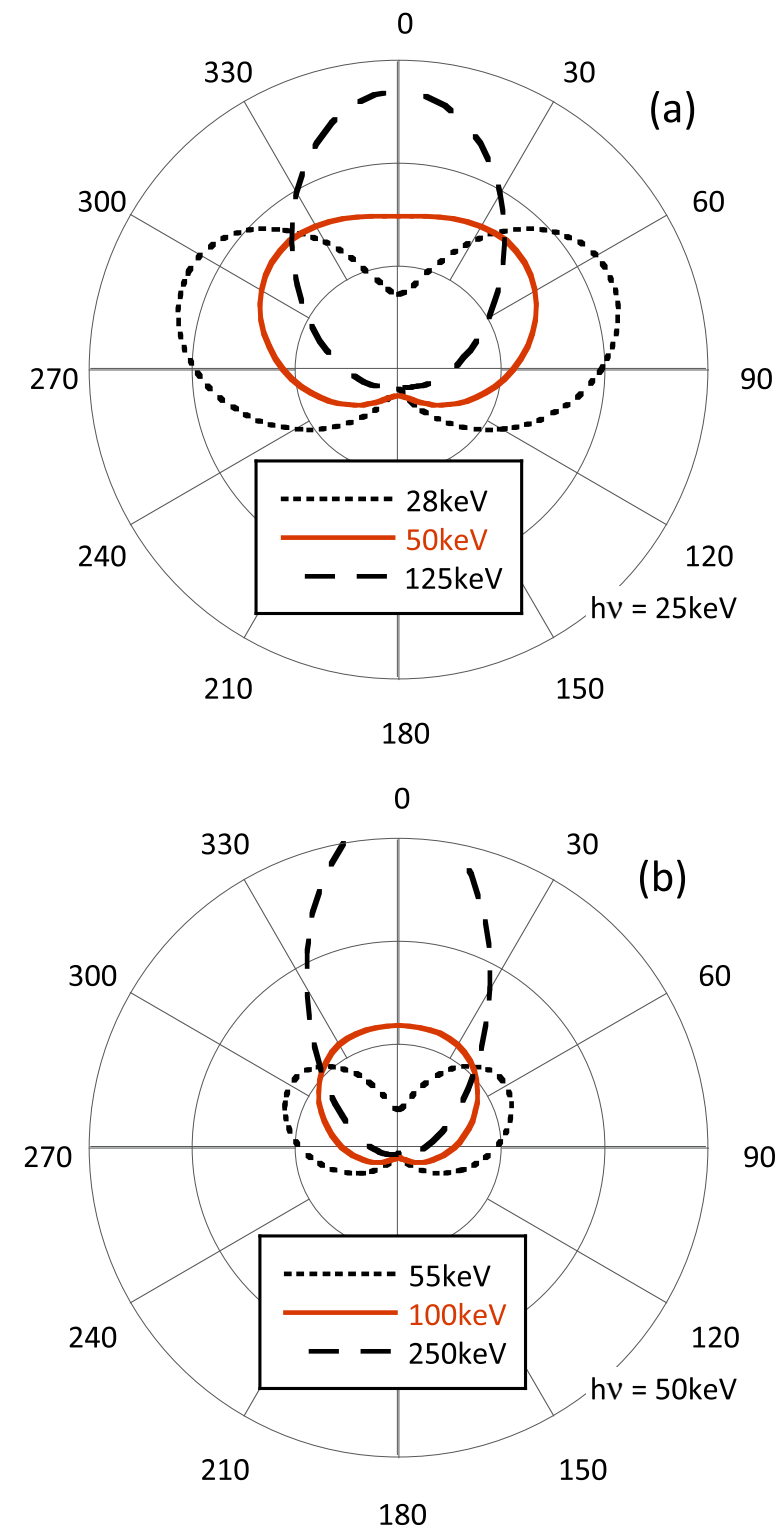

FIG. 2. (Color online) Expected x-ray emission as a function of angle relative to direction of an electron for various electron energies. The angular variation is given by the formula of [32] which is shown to match detailed partial wave calculations [34]. The radial axis has a maximum of $3 \times 10^{-50} \mathrm{ergs} \mathrm{sr}^{-1} \mathrm{~Hz}^{-1}$ atom ${ }^{-1}$ electron ${ }^{-1} \mathrm{~cm}^{-2}$ in both graphs. In (a) we look at $25 \mathrm{keV}^{-}$photon emission which is at the low end of what is transmitted through the filters but close to the peak of image plate sensitivity. We choose electron energies such that the photon energy is respectively $0.9,0.5$ and 0.2 of the electron energy. In (b) we do the same for $50 \mathrm{keV}$ photon emission.

common in the literature to describe the hard-x-ray spectrum from laser plasmas in terms of a single effective temperature. In this case, this seems to be a reasonable approximation. The data in Fig. 4(a) are fitted with a least-squares approach, and no attempt to add a second component of emission at a different effective temperature resulted in a better fit.

The correspondence between the slope of the x-ray spectrum from a solid foil and the fast-electron temperature requires some discussion. McCall [23], for example, pointed
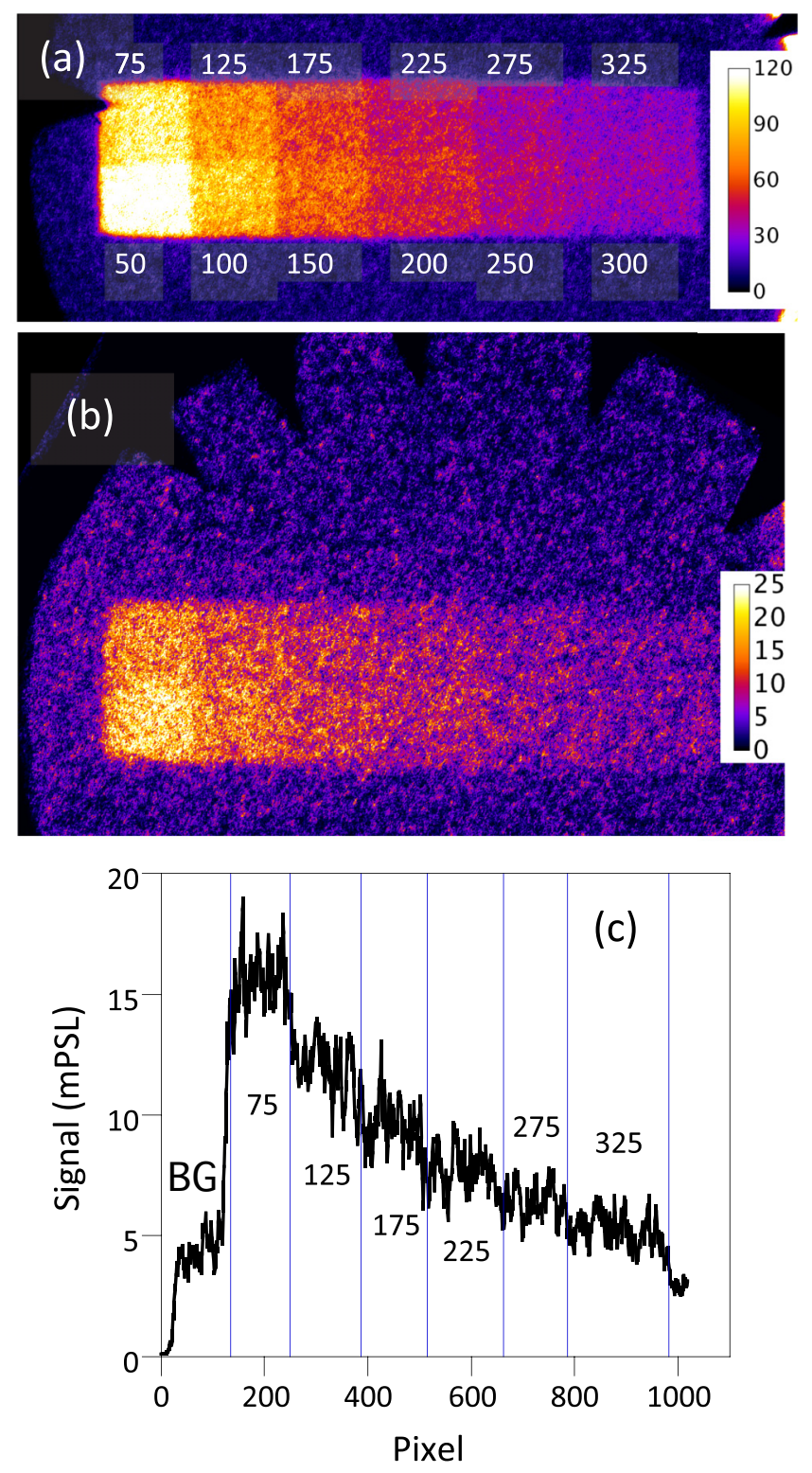

FIG. 3. (Color online) (a) Raw data image for channel 8 Ta target shot showing the clear demarcations of the $\mathrm{Pb}$ filters along with thickness in microns. The color bar is in MPSL units and the thinnest filter is slightly saturated on this scale to allow the reader to better see the filter boundaries. (b) Raw image of the data for channel 8 for a Ti target. (c) Averaged line out of the top row of filters, running from 75 to $325 \mu \mathrm{m} \mathrm{Pb}$ in steps of $50 \mu \mathrm{m}$ showing demarcations of the filters and thicknesses. The background (BG) level is also shown.

out that for measurements made at lower photon energies, the slope of photon emission does not necessarily correspond to the fast-electron temperature for one- and three-dimensional Maxwellian electron distributions. Our diagnostic does not measure the slope but integrates across all energies for each filter, and so, to test its validity, we have used a simple model based on the experimental observation that for monoenergetic electrons of energy, $E_{e}$, impinging on a foil, the bremsstrahlung energy emission spectrum scales as $I=C Z\left(E_{e}-E_{v}\right)^{\alpha}$, where $E_{v}$ is the photon energy, $\alpha$ is a constant close to unity (1.15 for Ti), and $Z$ is the atomic 

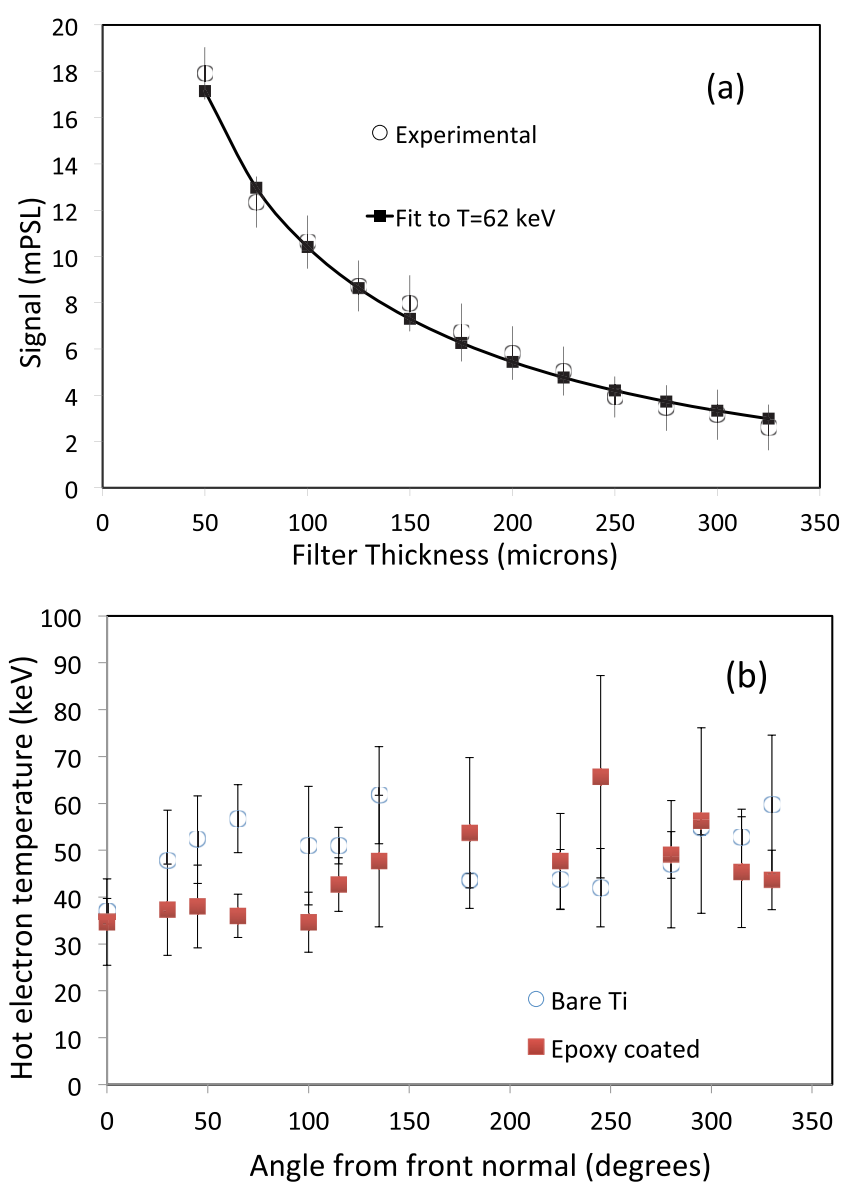

FIG. 4. (Color online) Sample data for $10 \mu \mathrm{m}$ Ti foil viewed at $30^{\circ}$ to front normal. The best fit is to $62 \mathrm{keV}$. (b) Fast-electron temperature determined for epoxy-coated foils and shots on bare foils. The values are averages over several shots (typically three to four) and the error bars show the standard error in the mean.

number of the foil material $[23,27,28]$. With this, we have predicted the signal that would be detected through our filter array for a given fast-electron distribution. By taking this predicted spectrum and working back to estimate temperature, we have determined that, for a purely exponential electron distribution (two-dimensional Maxwellian), the assumption of an exponential spectrum in analyzing the bremsstrahlung reproduces the assumed hot-electron temperature accurately. However, if the fast-electron distribution is one-dimensional, then there is a systematic underestimate of temperature by up to $20 \%$ at the highest values and an overestimate of similar magnitude if the electron distribution was fully three-dimensional. The analysis presented here assumes a two-dimensional Maxwellian.

In Fig. 4(b), we see the effective temperature determined at the full range of angles for both epoxy-coated and uncoated foils. For the epoxy-coated data, we have included the relatively small effect of absorption in the $1 \mathrm{~mm}$ epoxy layer, assuming the fast electrons do not penetrate far into the epoxy. As stated above, earlier work [22], with similar laser and target parameters, indicated that resonance absorption was the principal absorption mechanism. The temperature could be represented by the model of Wilks and Kruer,
$T_{h}(\mathrm{keV}) \approx 10\left[T_{c} I_{15} \lambda^{2}\right]^{1 / 3}[29]$, where $T_{c}$ is the background electron temperature in $\mathrm{keV}, I_{15}$ is the intensity in units of $10^{15} \mathrm{~W} \mathrm{~cm}^{-2}$, and $\lambda$ is the wavelength in $\mu \mathrm{m}$. Using this scaling and the data in Fig. 4, we estimate $T_{c}=100 \mathrm{eV}$ for the present run. For comparison, HYADES [30] simulation of the prepulses indicated a preformed plasma with density scale length $2.4 \mu \mathrm{m}$ and temperature $50 \mathrm{eV}$ at critical density prior to the main pulse interaction.

The averaged temperature from several shots for both types of target foil is shown in Fig. 4(b). For the epoxy-coated case, there is evidence that the inferred emission temperature is systematically lower than for uncoated foils when we look toward the front normal (from $270^{\circ}$ round to $90^{\circ}$ in Fig. 4 or from detectors $14 \mathrm{CW}$ round to 9 in Fig. 1). This can be related to the lack of refluxing of fast electrons and their anisotropy in hard-x-ray emission. As noted, we have attempted to fit the data from the detectors to a bi-Maxwellian distribution, but we have failed to find fits that are better than a single Maxwellian. We should note that this does not mean there is only one temperature of electrons. In addition to the bremsstrahlung from fast electrons penetrating the solid foil, there should be a so called "thermal" background emission coming from the plasma created on the laser-irradiated side. However, our $\mathrm{Pb}$ filters means that the detection system is effectively insensitive to $\mathrm{x}$ rays of energy below $25 \mathrm{keV}$, and this source of emission contributes little to the signal.

In Fig. 5(a), we can see a plot of the signal level on the image plate detected through the $50 \mu \mathrm{m} \mathrm{Pb}$ filter layer for both $10-\mu \mathrm{m}$-thick bare foils and $10-\mu \mathrm{m}$ foils coated in epoxy. The data are in terms of a raw signal on the image plate, but a slight normalization was applied to account for laser energy variations, although no shot was more than $6 \%$ from the average. Since the lead filter effectively cuts out emission below $25 \mathrm{keV}$, absorption in the epoxy has a minimal effect on signal.

In Fig. 5(a) there is a wide but distinct peak in the rear normal direction for the case of epoxy-coated targets, whereas the bare foil case is significantly more isotropic. The ratio of the signal to the rear and front target normal directions is $\sim 3.4$ for the epoxy-coated case. In addition, the overall emission is lower in the case of epoxy-coated targets for all directions. The data for $10 \mu \mathrm{m}$ epoxy-coated foils but taken through thicker $\mathrm{Pb}$ filters all the way to $300 \mu \mathrm{m}$ show a similar peak at the rear normal, although the ratio of the signal between the rear and front normal directions increases to nearly 5, as might be expected for a detection channel that samples higherenergy photons coming from the most energetic electrons. The solid line in Fig. 5(a) is a calculation made using the angular variation formulated originally by Kirkpatrick and Wiedmann [31,32], which has been shown to agree very well with tabulated partial wave calculations $[28,33]$. We have averaged it over a beam of electrons with energy distribution given by $f(E)=A \exp \left(-E / T_{h}\right)$, where $T_{h}=50 \mathrm{keV}$ is an effective fast-electron temperature, based on the analysis presented above. Spectral summation was carried out over all photon energies (out to $E_{\mathrm{ph}}=10 T_{h}$ ) using the bremsstrahlung spectrum approximation discussed above [23,27,28], which is folded with the exponential electron distribution to give an effectively time-integrated spectrum. The transmission for a $50 \mu \mathrm{m}$ lead filter [34,35] and the image plate response [24,25] 

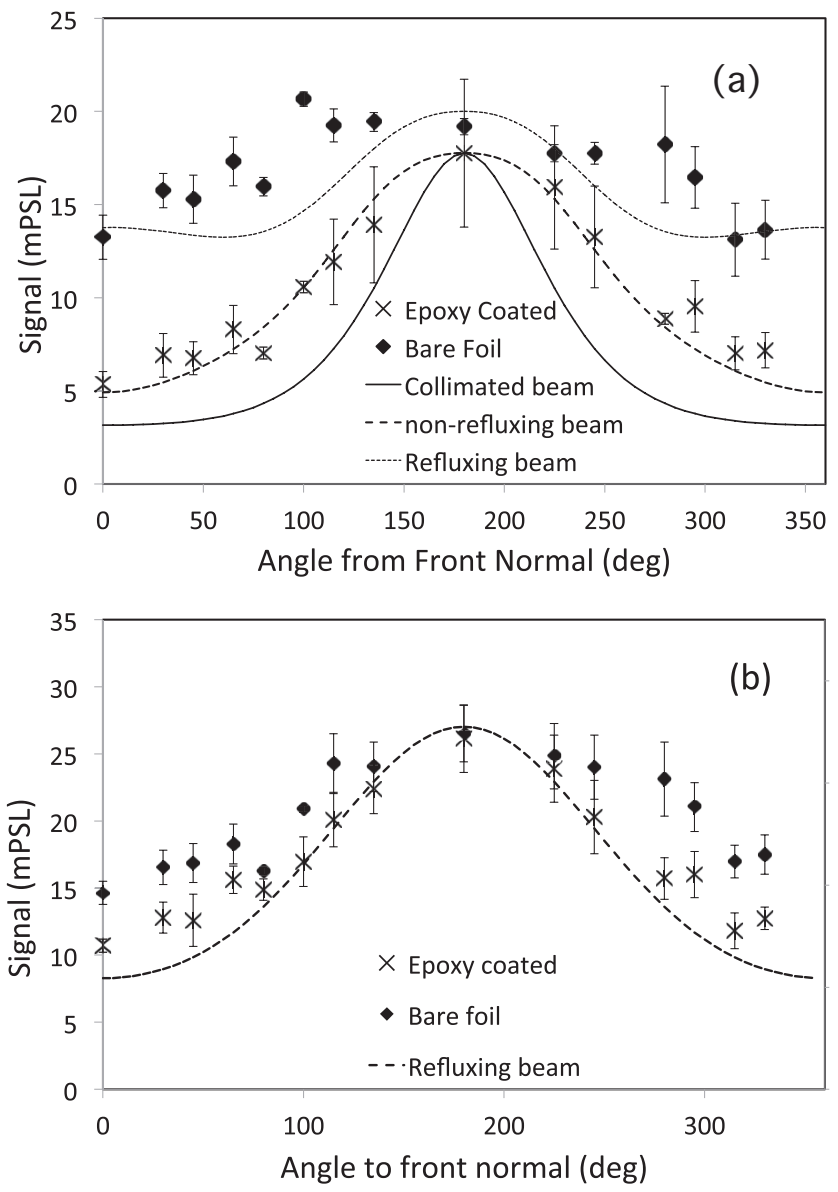

FIG. 5. (a) Signals through the $50 \mu \mathrm{m} \mathrm{Pb}$ filters for $10-\mu \mathrm{m}$-thick bare and epoxy-coated foils. The solid line is a prediction of the angular dependence for a collimated beam of electrons at a temperature of $50 \mathrm{keV}$ based on the angular dependence given by Kirkpatrick and Wiedmannn [31]. The dotted and dashed lines include broadening due to electron divergence as described in the text for bare foils and epoxy-coated foils, respectively. (b) Similar data for $50 \mu \mathrm{m}$ Ti with and without epoxy.

as a function of photon energy have both been folded in. The dashed and dotted lines are calculated by taking the angular emission model for a beam of electrons and folding with the electron divergence, as discussed next.

We have simulated our experiment using the ZEPHYROS 3D macroparticle hybrid simulation code [36,37]. This code injects an exponential distribution of fast electrons with a fixed effective temperature in a square pulse into a solid target. A resistivity model for $\mathrm{Ti}$ and a scattering model for fast electrons help to calculate the evolution of the current, magnetic and electric fields, and electron trajectories. We have used the code to track the momentum of each electron in the simulation. In doing so, we were able to generate temporal snapshots of the momentum direction of each macroparticle. With a postprocessor we were able to calculate the energy and direction with respect to each detector in the horizontal plane. We then used the spectral emission profile given by a corrected Bethe-Heitler approximation, again shown by Salvat et al. to be a good approximation to tabulated partial wave calculations [28,33]. By folding this with the normalized angular distribution of $\mathrm{x}$ rays calculated from KirkpatrickWiedmann [31,38], we were able to predict the emission as a function of angle. By averaging over snapshots every 200 fs until the fast electrons decay ( 2 ps), we generated a time-integrated prediction. Our results for $10 \mu \mathrm{m}$ foils can be seen in Fig. 5(a). The best results were found for an initial electron divergence full angle of $50^{\circ}$. As we can see, for the epoxy-coated targets there is a very good fit. This is not surprising, as a similar divergence was found in earlier work with the same laser under similar conditions [22]. In this respect, our hard-X-ray data that relate to the direction of fast-electron flow are complementary to and consistent with the $K-\alpha$ data in [22] that relate to the spatial location of fast-electron flow. Our inferred divergence is also consistent with the recent simulations of Ovchinnikov et al. [39], which were made for laser pulses of the same wavelength and a similar duration to ours. For the bare foils, the predicted emission is more isotropic, as seen in the experimental data. The simulation, however, does show slight dips centered around $90^{\circ}$ and $270^{\circ}$, which are the directions along the target surface. This is likely to be due to the simulation, which does not include target hydrodynamics, assuming a mirrorlike reflection of electrons from the rear surface of the foil. Nevertheless, the overall agreement between what is expected experimentally for the two cases and what is predicted is good and clearly illustrates the connection between the anisotropy and refluxing.

In Fig. 5(b), we see similar data but taken for $50 \mu \mathrm{m}$ $\mathrm{Ti}$ foils. In this case, we see anisotropy in both cases and a peak emission that is slightly higher than for the $10 \mu \mathrm{m}$ foil cases. This can be explained relatively simply. For a typical electron temperature of $50 \mathrm{keV}$, the range of an average energy electron is $30 \mu \mathrm{m}$ in Ti. This means that in the $10 \mu \mathrm{m}$ foils, the electrons reach the rear of the foil and reflux, thus enhancing the bremsstrahlung emission over the epoxy case, and also, since they change direction, it causes a more isotropic emission. For the $50 \mu \mathrm{m}$ foil, most electrons do not reach the rear of the foil, and so without refluxing the anisotropy is similar to the epoxy-coated case. For the thicker Ti foils, we might expect scattering to lessen the anisotropy, and indeed we can see that the ratio of the rear normal to the front normal signal drops to 2.4 for the $50 \mu \mathrm{m}$ foils. However, we can see that the simulated distribution determined with ZEPHYROS in the same way as for the $10 \mu \mathrm{m}$ case seems to fit quite well. Any additional scattering within the foil is clearly not enough to remove the anisotropy in the $\mathrm{x}$-ray emission totally.

It is of interest to see the effect of refluxing on other parameters within the ZEPHYROS simulations presented above. In Fig. 6, we can see the fast-electron density mapped. The simulations use an $n_{x}=40, n_{y}=125, n_{z}=125$ grid, where $x$ is the direction of propagation through the foil, left to right. The foil thickness is $10 \mu \mathrm{m}$. The divergence was set to 50 degrees full angle as for the simulations above. As in previous work [22] with the same laser where the $K-\alpha$ source size was determined to be a minimum of $70 \mu \mathrm{m}$, we have set the effective spot size to this value to account for the spreading of fast electrons out from the focal spot region before entering the foil.

The effect of refluxing in enhancing the fast electrons within the Ti foil is clear to see in Fig. 6, where there is roughly a 


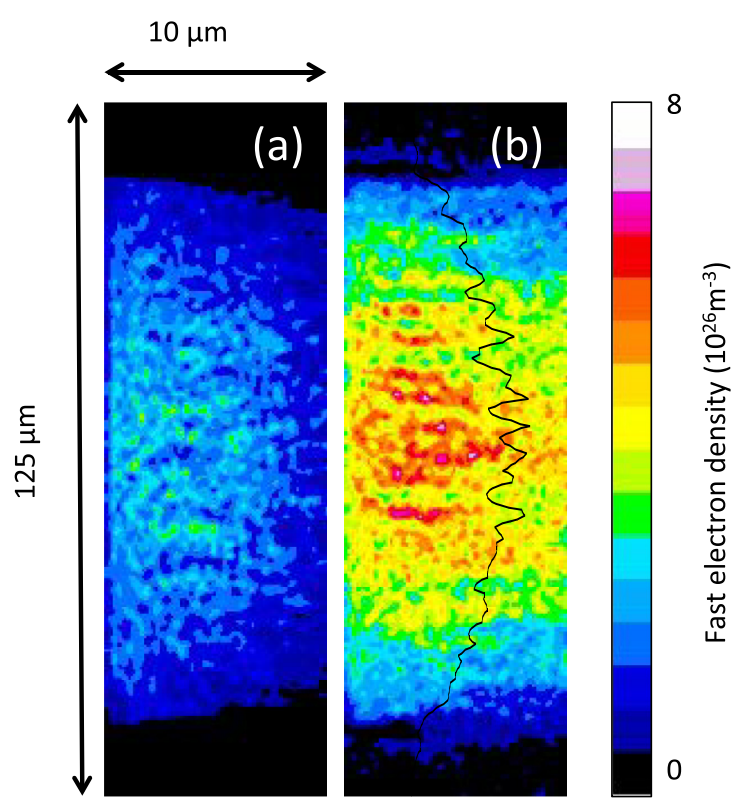

FIG. 6. (Color online) Fast-electron density for simulated $50 \mathrm{keV}$ effective electron temperature in Zephyros (a) without refluxing and (b) with refluxing. The data are taken in a snapshot at $0.8 \mathrm{ps}$, which is just at the end of the pulse in the simulation. In (b) the solid line shows a line out from the center of the foil indicating roughly $20 \%$ variations in electron density.

doubling of fast-electron density. For the refluxing case, we can see that there is also enhanced evidence of some filamentation in the fast-electron density with variations of about $20 \%$ across the electron beam. In Fig. 7, we see a clear difference again between the cases in which we consider the magnetic field. The small saturated regions in the refluxing case have
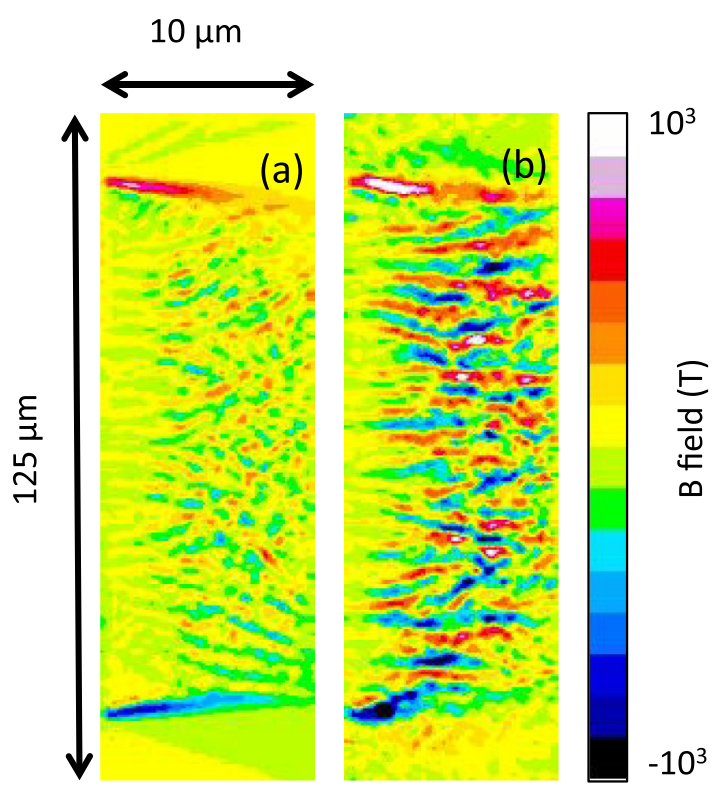

FIG. 7. (Color online) The $z$ component of the magnetic field at the center plane of the target for the same conditions as Fig. 6 (a) without refluxing and (b) with refluxing. magnetic fields reaching $1300 \mathrm{~T}$, while in the nonrefluxing case they reach $800 \mathrm{~T}$. The main difference, however, is in the strong appearance of interlacing "fingers" of magnetic fields in opposite directions. This is due to the interaction of two opposing streams of fast electrons. We expect in the nonrefluxing case that a Weibel-type instability can form due to the interaction of the fast electrons and the induced return current. The electrons reflected from the rear of the foil interact with the "incoming" fast electrons to enhance this in the refluxing case. The use of polarimetric reflection techniques to measure the magnetic field on the rear of laser-irradiated foils has been discussed by Chatterjee et al. [40], and this could in principle be applied to the bare foil case. However, experimental comparison of bare to coated foils in this case would require a rear surface layer that would allow the probe to pass unhindered but also absorb all the fast electrons reaching the rear of the foil. This would be a significant technical challenge.

In Fig. 8 we can see the background temperature in the simulation. We can see a clear difference in the two cases, with enhanced fingers of higher temperature in the refluxing case linked to the high fast-electron density regions caused by the instability in the magnetic field.

Anisotropic x-ray emission has been seen to occur before $[19,20]$, but it has generally been seen to occur at higher irradiance with electron temperatures in the strongly relativistic regime. In our case, the temperatures are somewhat lower but still relativistic $(\beta>0.5)$. Furthermore, in $[19,20]$, the peak in hard-X-ray emission is seen in the direction closer to specular reflection of the incident laser pulse. This has been connected to acceleration of electrons in the direction of the reflected pulse via the $\mathbf{J} \times \mathbf{B}$ mechanism, which is relevant at the higher intensities used. In other previous work [41] with much higher intensities and a more limited number of detectors, the

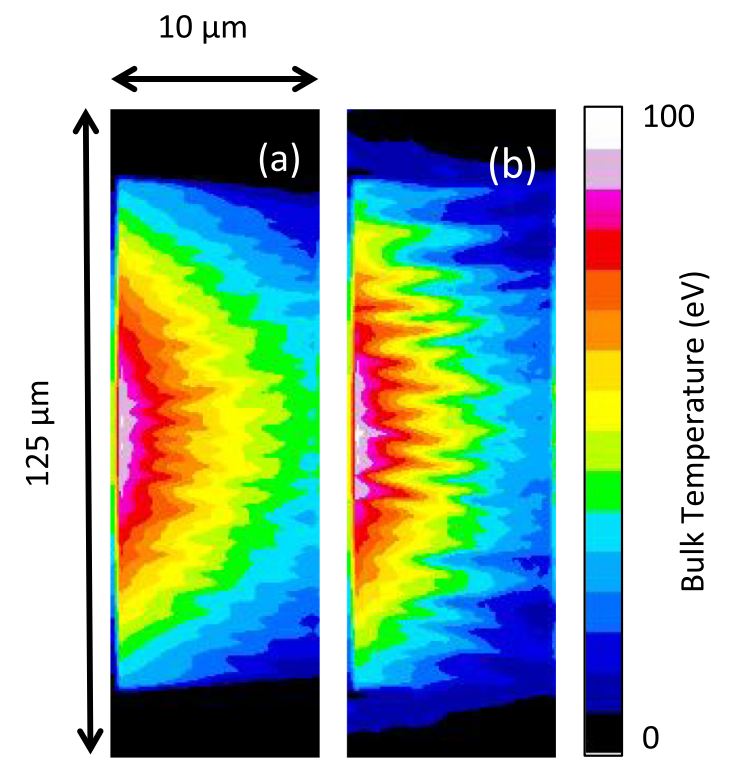

FIG. 8. (Color online) Background temperature at the center plane of the target for the same conditions as Fig. 6 (a) without refluxing and (b) with refluxing. 
hard-X-ray yield was seen to peak in a direction normal to the target rear, although no measurements were made from the front, laser-irradiated side. In that work, a top modest $Z$ layer (Al) covered the main Ag target, polystyrene was used to limit refluxing, and comparison was not made without the polystyrene. Work by Norreys et al. [42] showed a peak in $>10 \mathrm{MeV} \gamma$ rays that is between the target rear normal and the direction of the laser propagation. Again, measurements to the rear of the target only are presented, and in that case the mechanism for absorption of the laser energy to generate the required electron energies is likely to be quite different from our case. Finally, we note the paper by Li et al. [43], in which the fast electrons ejected from a target are detected. In that paper, the irradiance is similar to that used by us, but the pulse duration is much shorter ( $30 \mathrm{fs})$. At $45^{\circ}$ incidence on target they see electrons above $300 \mathrm{keV}$ predominately ejected normal to the target surface but with some evidence of electrons pushed along the target surface, a feature that becomes dominant at a higher angle of incidence $\left(70^{\circ}\right)$. In comparing this to the present work, it is worth noting that Li et al. detect only the higher-energy electrons that escape the target while our x-ray measurements are produced by the bulk of fast electrons within the target. It is possible that in our work, a small high-energy tail of electrons could exist, created by, for example, $\mathbf{J} \times \mathbf{B}$ heating, and that measurement of escaping fast electrons would show this. However, the $\mathrm{X}$-ray emission is, from our data, evidently dominated by a more modest temperature $(\sim 60 \mathrm{keV})$ population that is likely to be contained with the target.

In summary, we have made several key observations in our experiment. First, we have observed anisotropy in hard-x-ray emission at lower intensities than in previous work, at the lower limit of the relativistic regime with fast electrons below $100 \mathrm{keV}$ average energy. This may initially seem surprising but is less so when we consider that these electrons are still traveling at around half light speed, and relativistic transformation of the emission cross section is considerable, as seen in Fig. 2.

Secondly, the peak emission is directly in the direction normal to the rear of the target. This is due to resonance absorption being the dominant electron heating mechanism, which means that electron acceleration is primarily along the density gradient normal to the target. This is entirely consistent with operating in the weakly relativistic regime where mechanisms such as $\mathbf{J} \times \mathbf{B}$ acceleration are not yet dominant. In modeling the $\mathrm{X}$-ray emission angular profile, we have derived a value for the electron beam divergence that is in good agreement with predictions [39] and previous measurements [22] made with a different technique but under similar conditions.

Next, the measured effective temperature of the fast electrons has been shown to be more isotropic than the x-ray emission intensity. This is consistent with the observation that a single Maxwellian approximation fits the data better than a two-temperature approximation. Finally, we have made observations directly linking angular distribution of hard-X-ray emission from intense laser-plasma interactions with electron refluxing. We have, in fact, been able to switch the anisotropy on and off. The significance of this work is that electron dynamics can be revealed through hard-x-ray emission and understanding this is key to understanding and developing applications of intense laser-solid interactions such as particle beam generation.

This work was supported by the UK Engineering and Physical Sciences Research Council (Grants No. EP/C003586/1, No. EP/G007462/01, No. EP/I029206/1, and No. EP/L013975/1).
[1] W. L. Kruer, The Physics of Laser Plasma Interactions (Westview, Boulder, CO, 2003).

[2] A. Rousse, P. Audebert, J. P. Geindre, F. Fallies, J. C. Gauthier, A. Mysyrowicz, G. Grillon, and A. Antonetti, Phys. Rev. E 50, 2200 (1994).

[3] C. Reich, P. Gibbon, I. Uschmann, and E. Forster, Phys. Rev. Lett. 84, 4846 (2000).

[4] D. C. Eder, G. Pretzler, E. Fill, K. Eidmann, and A. Saemann, Appl. Phys. B 70, 211 (2000).

[5] K. B. Wharton et al., Phys. Rev. Lett. 81, 822 (1998).

[6] T. Feurer et al., Phys. Rev. E 65, 016412 (2001).

[7] J. D. Kmetec, C. L. Gordon, J. J. Macklin, B. E. Lemoff, G. S. Brown, and S. E. Harris, Phys. Rev. Lett. 68, 1527 (1992).

[8] M. Borghesi, J. Fuch, S. V. Bulanov, A. J. Mackinnon, P. K. Patel, and M. Roth, Fusion Sci. Technol. 49, 412 (2006).

[9] S. Fourmaux, C. Serbanescu, R. E. Kincaid, Jr., A. Krol, and J. C. Kieffer, Appl. Phys. B 94, 569 (2009).

[10] H. S. Park et al., Phys. Plasmas 13, 056309 (2006).

[11] M. Nishikino et al., Rev. Sci. Instrum. 81, 026107 (2010).

[12] S Chen et al., Phys. Plasmas 14, 102701 (2007).
[13] J. Myatt, W. Theobald, J. A. Delettrez, C. Stoeckl, M. Storm, T. C. Sangster, A. V. Maximov, and R. W. Short, Phys. Plasmas 14, 056301 (2007).

[14] M. N. Quinn, X. H. Yuan, X. X. Lin, D. C. Carroll, O. Tresca, R. J. Gray, M. Coury, C. Li, Y. T. Li, C. M. Brenner, A. P. L. Robinson, D. Neely, B. Zielbauer, B. Aurand, J. Fils, T. Kuehl, and P. McKenna, Plasma Phys. Controlled Fusion 53, 025007 (2011).

[15] V. T. Tikhonchuk, Phys. Plasmas 9, 1416 (2002).

[16] P. Neuymayer et al., Phys. Plasmas 17, 103103 (2010).

[17] G. Nersisyan et al., Phys. Rev. E 85, 056415 (2012).

[18] S. Buffechoux et al., Phys. Rev. Lett. 105, 015005 (2010).

[19] F. Zamponi et al., Phys. Rev. Lett. 105, 085001 (2010).

[20] S. Düsterer et al., Contrib. Plasma Phys. 41, 171 (2001).

[21] T. Dzelzainis et al., Laser Particle Beams 28, 451 (2010).

[22] M. Makita et al., Phys. Plasmas 21, 023113 (2014).

[23] G. H. McCall, J. Appl. Phys. 15, 823 (1982).

[24] T. Döppner et al., Rev. Sci. Instrum. 83, 10E508 (2012).

[25] A. L. Meadowcroft, C. D. Bentley, and E. N. Stott, Rev. Sci. Instrum. 79, 113102 (2008).

[26] K. J. A. McKeever, Ph.D. thesis for Queen's University of Belfast 2014. 
[27] E. Storm, Phys. Rev. A 5, 2328 (1972).

[28] F. Salvat, J. M. Fernández-Varea, J. Sempau, and X. Llovet, Radiat. Phys. Chem. 75, 1201 (2006).

[29] S. C. Wilks and W. L. Kruer, IEEE J. Quantum Electron. 33, 1954 (1997).

[30] J. T. Larsen and S. M. Lane, J. Quantum Spectrosc. Radiat. Transf. 51, 179 (1994).

[31] P. Kirkpatrick and L. Wiedmann, Phys. Rev. 67, 321 (1945).

[32] Z. J. Ding, R. Shimizu, and K. Obori, J. Appl. Phys. 76, 7180 (1994).

[33] L. Kissel, C. A. Quarles, and R. H. Pratt, At. Data Nucl. Data Tables 28, 381 (1983).

[34] NIST Standard Reference Database 126: S. M. Seltzer, Radiat. Res. 136, 147 (1993).
[35] NIST Standard Reference Database 126: Int. J. Appl. Radiat. Isotopes 33, 1269 (1982).

[36] S. Kar, A. P. L. Robinson, D. C. Carroll, O. Lundh, K. Markey, P. McKenna, P. Norreys, and M. Zepf, Phys. Rev. Lett. 102, 055001 (2009).

[37] A. P. L. Robinson, M. H. Key, and M. Tabak, Phys. Rev. Lett. 108, 125004 (2012).

[38] E. Acosta, X. Llovet, E. Coleoni, J. A. Riveros, and F. Salvat, J. Appl. Phys. 83, 6038 (1998).

[39] V. M. Ovchinnikov et al., Phys. Rev. Lett. 110, 065007 (2013).

[40] G. Chatterjee et al., Phys. Rev. Lett. 108, 235005 (2012).

[41] C. D. Chen et al., Phys. Plasmas 20, 052703 (2013).

[42] P. Norreys et al., Phys. Plasmas 6, 2150 (1999).

[43] Y. T. Li et al., Phys. Rev. Lett. 96, 165003 (2006). 